\title{
Hypoxia induced ferritin light chain (FTL) promoted epithelia mesenchymal transition and chemoresistance of glioma
}

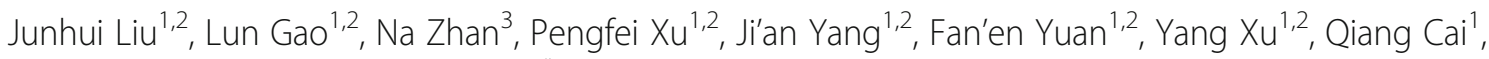
Rongxin Geng ${ }^{1,2}$ and Qianxue Chen ${ }^{1 *}$

\begin{abstract}
Background: Hypoxia, a fundamental characteristic of glioma, is considered to promote tumor malignancy by inducing process of epithelial mesenchymal transition (EMT). Ferritin Light Chain (FTL) is one of the iron metabolism regulators and is overexpressed in glioma. However, relationship between hypoxia and FTL expression and its role in regulating EMT remains unclear.

Methods: Immunohistochemistry (IHC), western blot and public datasets were used to evaluate FTL level in glioma. Wound healing, transwell assays, CCK8, annexin V staining assay were used to measure migration, invasion, proliferation and apoptosis of glioma cells in vitro. Interaction between HIF1A and FTL was assessed by luciferase reporter and Chromatin immunoprecipitation (ChIP) assays. Subcutaneous xenograft model was established to investigate in vivo growth.

Results: FTL expression was enriched in high grade glioma (HGG) and its expression significantly associated with IDH1/2 wildtype and unfavorable prognosis of glioma patients. FTL expression positively correlated with HIF1A in glioma tissues and obviously increased in U87 and U251 cells under hypoxia in a time-dependent manner. Mechanistically, HIF-1a regulates FTL expression by directly binding to HRE-3 in FTL promoter region. Furthermore, we found that knockdown FTL dramatically repressed EMT and reduced migration and invasion of glioma by regulating AKT/GSK3 $\beta / \beta$-catenin signaling both in vitro and in vivo. Moreover, our study found downregulation FTL decreased the survival rate and increased the apoptosis of glioma cells treated with temozolomide (TMZ). FTL expression segregated glioma patients who were treated with TMZ or with high MGMT promoter methylation into survival groups in TCGA dataset. Patients with methylated MGMT who had high FTL expression presented similar prognosis with patients with unmethylated MGMT.
\end{abstract}

Conclusion: Our study strongly suggested that hypoxia-inducible FTL was a regulator of EMT and acted not only as a prognostic marker but also a novel biomarker of response to TMZ in glioma.

Keywords: Ferritin light chain, Hypoxia, Epithelial mesenchymal transition, Chemoresistance, Glioblastoma, Prognosis

\footnotetext{
* Correspondence: chenqx666@whu.edu.com

'Department of Neurosurgery, Renmin Hospital of Wuhan University, No.238, jiefang Road, Wuchang District, Wuhan 430060, Hubei Province, China

Full list of author information is available at the end of the article
}

C C The Author(s). 2020 Open Access This article is licensed under a Creative Commons Attribution 4.0 International License, which permits use, sharing, adaptation, distribution and reproduction in any medium or format, as long as you give appropriate credit to the original author(s) and the source, provide a link to the Creative Commons licence, and indicate if changes were made. The images or other third party material in this article are included in the article's Creative Commons licence, unless indicated otherwise in a credit line to the material. If material is not included in the article's Creative Commons licence and your intended use is not permitted by statutory regulation or exceeds the permitted use, you will need to obtain permission directly from the copyright holder. To view a copy of this licence, visit http://creativecommons.org/licenses/by/4.0/ The Creative Commons Public Domain Dedication waiver (http://creativecommons.org/publicdomain/zero/1.0/) applies to the data made available in this article, unless otherwise stated in a credit line to the data. 


\section{Background}

Glioma originated from the neuroectoderm and accounts for approximately $81 \%$ of primary malignant brain tumors [1]. Glioma is graded I-IV according to the 2016 World Health Organization classification of central nervous system tumors. Glioblastoma is the most common and malignant subtype and the dismal 2-year and 5-year survival rate are $<40$ and $<10 \%$,respectively [2]. Low grade gliomas (WHO I-II) usually progress to higher grade and eventually have poor outcomes despite treating with standard care (surgical resection combined with postoperative radiotherapy and chemotherapy) [3]. Therefore, it is urgent to understand the key molecular mechanisms in the malignancy progression of glioma to develop more effective treatments.

Hypoxia is a common pathological feature in glioma. Increasing grade of gliomas correlate with an increase in absence of oxygen [4]. Chronic hypoxia often leads to necrosis in tumor tissues, which is one of the most distinct characteristics of glioblastoma. Hypoxia microenvironment promotes glioma cells aggressive phenotype by upregulating hypoxia-inducible factor (HIF) family [5-7].HIF1A is highly expressed in glioblastoma and significantly correlated with IDH1/2 mutation [8]. Moreover, previous studies have demonstrated that hypoxia environment could induce the epithelialmesenchymal transition (EMT) during the progression of glioma by regulating several pathways, such as Wnt/ $\beta$-catenin [9], transforming growth factor $\beta$ (TGF- $\beta$ ) [10] and Sonic Hedgehog ( $\mathrm{SHH})$ pathway [11]. This phenotype transition facilitated glioma cells easier to infiltrate the adjacent brain tissues and more resistance to chemo/radiotherapy [12]. However, the specific mechanism underlying which hypoxia promoting glioma malignancy remains to be further illustrated.

Ferritin was consisted of 24 units of heavy chain (FTH) and light chain (FTL). FTL had been widely recognized as one of the iron metabolism regulators for a long time. While in recent years, a growing number of studies have revealed the close relationship between FTL and tumor malignancies [13-16]. It was revealed that FTL could be used as a biomarker to discriminate benign and malignant tumors, and to predict the prognosis of patients with tumors $[17,18]$. Besides, FTL was found to be overexpressed in various malignant tumors, and played a crucial role in regulating malignancy progress of cancers $[19,20]$. Recent studies revealed that FTL could be upregulated on the post-transcriptional level by hypoxic conditions. Alveolar macrophages had 2.5 folds content of FTL when cells were exposed to hypoxia. Similar results were also observed in another study conducted by Sammarco et. HEK 293 cells were cultured in 1\% oxygen and the results showed that FTL and FTH were differentially upregulated $[21,22]$. These results suggested that
FTL might be regulated under the hypoxia environment. While the effect of hypoxic environment on FTL expression and its regulation in process of glioma malignancy have not been well investigated so far.

In this study, we found that FTL was higher in HGG than in Low grade glioma (LGG). High FTL expression closely associated with wildtype IDH $1 / 2$ and poor prognosis. We showed, for the first time that FTL was a hypoxia-responsive gene that significantly elevated under hypoxia in a time-dependent manner in U87 and U251 cells. Further analysis revealed that HIF-1A regulates FTL expression by directly binding to HRE-3 in the FTL promoter region. Functionally, we showed that FTL induced the EMT and promoted migration, invasion and chemo-resistance of glioma both in vitro and in vivo. Mechanistically, oncogenic role of FTL was functioned by regulating $\mathrm{AKT} / \mathrm{GSK} 3 \beta / \beta$-catenin signaling.

\section{Materials and methods Clinical samples}

Glioma tissues were obtained from the department of neurosurgery in Renmin hospital of Wuhan University from July 2015 to July 2018.A total of 142 paraffinembedded glioma tissue were used for immunohistochemical staining. For western blot,28 glioma frozen tissues (stored at $-80^{\circ} \mathrm{C}$ ) of different grades were evaluated. Details of clinical information for all patients was presented in Table S1. None patients received any chemo- or radiotherapy before surgery. All patients signed informed consents and this study received the approval of the Ethics Committee of Renmin Hospital of Wuhan University (approved number: 2012LKSZ (010) $\mathrm{H})$.

\section{Immunohistochemical (IHC) staining}

The paraformaldehyde-fixed paraffin tissue microarray that contained 142 glioma tissues was used. The microarray was incubated with a primary anti-FTL monoclonal antibody (Abcam, USA: Ab109019) overnight at $4{ }^{\circ} \mathrm{C}$. Images were captured using an Olympus BX40 microscope (Tokyo, Japan). Two individuals were separately responsible for the assessment of the results. The result was primarily based on the strength of staining and the number of positive cells.10 high magnification fields were randomly selected for observation. Positive staining rate was scored as: 0 points for less than $5 \%, 1$ point for $5-25 \%, 2$ points for $26-50 \%, 3$ points for $51-75 \%$ and 4 points for $75 \%$. Besides, the classification of the strength of staining was followed: non-staining is 0 points, light yellow is 1 point, brown yellow is 2 points, and brown is 3 points. Finally, multiply the two scores to get the final score which would be graded into 4 grades: negative $(0$ point), weakly positive (1-4 points), positive(5-8 points) and strongly positive(9-12 points). Final score less than 
5 was defined as low expression and IHC score 5-12 was considered as high expression.

\section{Immunofluorescence staining}

Cells were fixed with $4 \%$ paraformaldehyde for $15 \mathrm{~min}$ and penetrated with $0.5 \%$ Triton X-100 (made in PBS) at room temperature for $10 \mathrm{~min}$. Then slides were washed with PBS 3 times. 1\%BSA was added dropwise on the slides at room temperature for $30 \mathrm{~min}$. Then we added a sufficient amount of the diluted primary antibody and placed it in a wet box, incubate at $4{ }^{\circ} \mathrm{C}$ overnight. Incubation with secondary antibody (Antgene, Wuhan, China) was performed in a wet box at $37^{\circ} \mathrm{C}$ for $1 \mathrm{~h}$ under dark conditions. DAPI (ANT046, Antgene) was added in the dark for $5 \mathrm{~min}$. Finally, slides were observed under a fluorescence microscope (Olympus BX51, Japan) to acquiring images.

\section{Cells, cell culture and transfection}

Two human glioblastoma-derived cancer lines, U251 and U87 were purchased from the Cell Bank Type Culture Collection of the Chinese Academy of Sciences (Shanghai, China). Cell lines were identified by Procell Life Science\&Technology Co.,Ltd. (Wuhan, China). Cell lines were all cultured at $37{ }^{\circ} \mathrm{C}$ under a humidified atmosphere of $5 \% \mathrm{CO}_{2}$ by using Dulbecco's modified Eagle's medium (DMEM) supplemented with 10\% fetal bovine serum (FBS) (Gibco, Invitrogen, Carlsbad, CA, USA). All cell lines were cultured without antibiotics. Short hairpin RNA (shRNA) targeted FTL and a scramble shRNA were purchased from Genechem Co., Ltd. (Shanghai, China). The target sequences against human FTL(5'-GGCGA GTATCTCTTCGAAA-3') and scrambled shRNA(5'-TTTCGAAGA GATACTCGCC-3') were cloned into the GV248 lentiviral vector. U251 and U87 cells were transfected with Lentivirus for $72 \mathrm{~h}$ and treated with puromycin $(4 \mu \mathrm{g} / \mathrm{ml})$ for 7 days. The specific small interfering RNA (siRNA) for FTL (siG143101050 18-1-5),HIF1A(siG0811494537-1-5),

CTNNB1(siB08220115 751-1-5),

HIF2A (siG170217101514-1-5) and scramble siRNAs were purchased from RiboBio (Guangzhou, China). Cells were cultured in a 6-well plate and transfected with lip2000(Invitrogen, Carlsbad, CA, USA) following the instructions of the manufacturer. After $48 \mathrm{~h}$ of transfection, the cells were used for subsequent experiments. FTL overexpression plasmid and a blank pcDNA3.1 vector were constructed. $2 \times 10^{5}$ cells were cultured in a 6 well plate and transfected with $2 \mathrm{ng}$ plasmid using lip3000(Invitrogen, Carlsbad, CA, USA) following the instructions of the manufacturer. The cells were harvested after transfected for $48 \mathrm{~h}$ for further experiments. For in vitro hypoxia experiments, cells were cultured in a consistent $1 \% \mathrm{O}_{2}$ hypoxic condition. The hypoxia mimetic cobalt chloride $\left(\mathrm{CoCl}_{2}\right)$ (Sigma, NO.232696) was dissolved in sterile PBS and the final concentration of $\mathrm{CoCl}_{2}$ in the medium was $200 \mathrm{uM}$. To monitor resistance to temozolomide (TMZ), the U87 and U251 cells were treated with TMZ (Selleck, NO.S1237) at various concentrations for $24 \mathrm{~h}$.

\section{Bioinformatics analysis}

To clarify the expression and prognostic role of FTL in gliomas, we used the Gliovis database (http://gliovis. bioinfo.cnio.es/) and the UCSC Xena platform (http:// xena.ucsc.edu/). Normalized RSEM gene-level RNAseq and corresponding clinical data of The Cancer Genome Atlas (TCGA),Rembrandt and IVY dataset were downloaded from Gliovis. Specific information on postoperative treatments (chemo/ radiotherapy) of glioma patients was downloaded from UCSC Xena platform. Besides, normalized mRNA expression (mRNA-array_693, (batch 1)) and clinical data were downloaded from Chinese Glioma Genome Atlas (CGGA). Low grade glioma was defined as WHO grade I-II and High grade glioma was defined as WHO III-IV according to the 2016 World Health Organization classification of central nervous system tumors [23].

\section{RNA isolation and RT-PCR}

Total RNA of U251 and U87 cell lines were extracted using TRIzol regent (Invitrogen). We used PrimeScript RT reagent kit with gDNA Eraser (Takara, Tokyo, Japan) to prepared for cDNA and real-time PCR was performed by using SYBR Green II Mixture (TaKaRa) according to the manufacturer's protocol. GAPDH was used for normalization and the comparative $\mathrm{Ct}$ method $(\Delta \Delta \mathrm{Ct})$ was used to evaluate mRNA expression. The specific primer pairs were as follows: GAPDH (internal control gene) primer (forward primer, 5' -ACAACTTTGGTATC GTGGA AGG-3'; reverse primer, 5'-GCCATCACGC CACAGTTTC-3'); FTL primer (forward primer, 5' CAGCCTGGTCAATTTGTACCT-3'; reverse primer, 5'-GCCAATTCG CGGAAGAAGTG-3').

\section{Western blot}

U251 and U87 were lysed in a modified RIPA buffer (NO.P0013B, Beyotime Biotechnology, China) on ice for about $30 \mathrm{~min}$, then centrifuged at 12,000 rpm for 15 min. For frozen glioma tissues, we added $1 \mathrm{ml}$ of RIPA lysate per $100 \mathrm{mg}$ of tissues. The concentration of the sample was quantitatively determined by BCA protein assay. The lysate was mixed with loading buffer after heated at $100^{\circ} \mathrm{C}$ for 5 mins.In brief, equal protein amount was loaded on $8-12 \%$ SDS-PAGE and then transferred to a nitrocellulose membrane. Next PVDF membrane was blocked in 5\% non-fat milk for $1 \mathrm{~h}$ and incubated with primary antibody at $4{ }^{\circ} \mathrm{C}$ overnight. 
Secondary antibodies (Antgene,Wuhan,Chian,1:10000) were used to incubate the membrane in shade environment at room temperature for $1 \mathrm{~h}$. The membranes were visualized with Odyssey (LI-COR biosciences, USA). Primary antibodies used were presented in Table S2.Western blot analysis were repeated three times.

\section{Wound healing and transwell assay}

Cells were seeded in a 6-well plate and cultured for a certain time to reach a $>90 \%$ confluence. The sterile pipette tip was used to scratch a linear wound and serum free DMEM was added for further culturing. Wound healing images were captured using an inverted microscope (Olympus BX51, Japan) and ImageJ software was used to analyze relative area of wound closed. For transwell assay, appropriated glioma cells were seeded into the upper well (Corning, USA) precoated with Matrigel (R\&D, USA). The lower chamber was filled with $600 \mu \mathrm{l}$ of DMEM containing 10\% FBS. Transwell chambers were placed in an incubator $\left(37^{\circ} \mathrm{C}, 5 \% \mathrm{CO}_{2}\right)$ for $24 \mathrm{~h}$. Cells in the upper chamber were fixed with $4 \%$ paraformaldehyde for $15 \mathrm{mins}$, stained with $0.1 \%$ crystal violet for $15 \mathrm{mins}$ and counted under an inverted microscope (Olympus BX51, Japan). We randomly selected 6 fields to count the number of invading cells in each set of experiments. All assays were repeated 3 times.

\section{Clone formation and cell count kit-8(CCK8) assay}

1000 glioma cells were counted and seeded in 6-well plates. Cells were cultured with DMEM supplemented with 10\% FBS and then Temozolomide was used to treat cells for 24 h.Cells were continued to be cultured in complete medium for about 10 days. Clones that contained more than 50 cells were scored. The clone formation rate was defined as the number of scored clones divided by the total cells seeded. For CCK8 assay,3000 cells were resuspended in $100 \mu \mathrm{l}$ DMEM supplemented with $10 \%$ FBS and then added to a 96 well plate. Various concentrations of temozolomide were added. Cell proliferation was investigated using CCK8(Dojindo Molecular Technologies, USA) according to the manufacturer's instruction.

\section{Flow cytometric analysis}

Cells were seeded in a 6-well plate and treated with temozolomide $(400 \mu \mathrm{M})$ for $72 \mathrm{~h}$. Annexin V-PE/7- ADD kit (Becton Dickinson, USA) were used to measure the apoptosis of glioma cells. All operations were carried out according to the manufacturer's instruction. In briefly, cells were harvested and washed three times with PBS. Then cells were stained with Annexin V-PE/7- ADD for 10 min under dark conditions. The apoptosis of samples was measured by FACS Calibur flow cytometer (BD
Biosciences, USA). Early apoptosis and late apoptosis were summed and the total apoptosis rate was calculated.

\section{TUNEL assay}

In Situ Cell Death Detection Kit was used to detected DNA fragmentation in apoptotic cells in xenografts according to the manufacturer's protocol (Roche). In short, sections were deparaffinised at $60^{\circ} \mathrm{C}$ for $20 \mathrm{~min}$ on a heat block and then incubated in xylene $(3 \times 5 \mathrm{~min})$. Tissue was then rehydrated by washing in graded alcohol, 3 min for each, after which they were rinsed in PBS three times. After treated with $0.1 \%$ Triton X-100 and Proteinase $\mathrm{K}$, the sections were incubated with TUNEL reaction mixture and incubated with converter-POD. Subsequently, DAB was used to stain slides. An Olympus BX51 microscope (Olympus) was used for image acquisition.

\section{Luciferase assays}

To investigate the role of interaction between HIF1A and FTL, we constructed FTL promoter-driven luciferase reporter plasmids and transfected into U87 and U251 cells. Cells were pretreated with si-HIF1A or scramble for $48 \mathrm{~h}$ and then exposed to $1 \% \mathrm{O}_{2}$ for 24 h.To further determine the direct binding between HIF1A and HREs in FTL promoter, we constructed mutant luciferase plasmid with ablation of HREs on FTL promoter by changing $5^{\prime}-\mathrm{GCGTG}-3^{\prime}$ to $5^{\prime}-\mathrm{GCTCT}-3^{\prime}$ and then co-transfected with plasmid containing the Renilla luciferase gene. Firefly luciferase activities were normalized using Renilla luciferase. Besides, TCF/LEF luciferase reporter (No.11542ES03) assay was performed using the Dual Luciferase Reporter Gene Assay Kit (Yeasen Biotech Co., Ltd. Shanghai, China) according to the manufacturer's protocol. All experiments were repeated three times.

\section{Chromatin immunoprecipitation (ChIP)}

ChIP was performed to explore the potential binding between the promoter region of FTL and HIF1A in glioma cells. U87 cells were incubated under the hypoxic condition for 24h.Antibody against HIF1A was purchased from cell signaling technology (Danvers, USA). Precipitated DNAs that contained FTL fragments were then amplified using quantitative PCR. The sequences of three primers used to detect HREs in FTL promoter were as follows:1\# (F): 5'-CGCAGGGCTTCTCTTT GTGG-3', (R): 5' - TGAACAGTGTCTCTGAAGTTGC C -3'; 2\# (F): 5' - CCACAACGCAGGGCTTCT C-3', (R): $5^{\prime}$-TTTGGAGACAACTCACAGACTTCG -3'; 3\# (F): 5' - CGCAGG GCTTCTCTTTGTGG -3', (R): 5' GGAGTGGAAATGGGGAGGAATG - 3'. 


\section{In vivo experiments}

All nude mice were purchased from Shulaibao (Wuhan, China) Biotechnology Co., Ltd. Animal feeding and experimental operations were in line with the guidelines of the Animal Ethics Committee of Wuhan University People's Hospital. Stably transfected U87 cells that were growing in the logarithmic phase were prepared. Cells were resuspended in PBS at a concentration of $5 \times 10^{6}$ cells $/ 100 \mu \mathrm{L}$ and then subcutaneously injected into the armpits of 5-week-old Balb/c nude mice. For in vivo temozolomide (TMZ) treatment, Six nude mice were randomly divided into two groups. Starting on day 5 , the mice were injected intraperitoneally with TMZ $(50 \mathrm{mg} /$ $\mathrm{kg}$ ) for 5 days. After subcutaneous implantation, the condition of the nude mice was observed daily. Recorded dynamic changes in the size of subcutaneous xenografts (longest diameter * shortest diameter $2 / 2$ ). All nude mice were sacrificed on the 29th day after transplantation and the tumors were weighted.

\section{Statistical analysis}

Data were presented as mean values \pm standard deviation (SD) from at least three experiments. Student's ttest was used to analyze the differences between two groups. One-way analysis of variance (ANOVA) was used for the comparison among three or more groups and the student-Newman-Keuls (SNK) method was used for post-analysis. Patients were divided into high and low groups according to the $50 \%$ cutoff point of FTL expression and Kaplan-Meier survival analysis was used to analyzed significance between groups. Univariate and multivariates Cox regression analysis was assessed by SPSS.21 (IBM, New York) software. Graphs production were performed by GraphPad Prism 5.0 software (GraphPad Software, Inc., La Jolla, USA). A $p$ value of less than 0.05 was considered as statistical significance.

\section{Results}

FTL is overexpressed and associates with prognosis in high grade glioma (HGG)

Our previous study revealed that FTL was elevated in glioma tissues when compared with non-tumor brain tissues by RT-PCR in a small glioma cohort [13]. While FTL expression in gliomas and its relationship with tumor malignancy remained poorly understood. In this study, we found that mRNA expression of FTL was enriched in HGG than in LGG using RNAseq data from three public datasets (Fig. 1a, Figure S1A). Moreover, FTL protein level was also elevated in HGG compared with that of LGG detected by IHC staining and WB (Fig. 1c-f). To explore the correlation between FTL and glioma aggressiveness, we compared FTL expression in different IDH1/2 status. FTL expression was significantly higher in IDH1/2 wildtype gliomas when compared with
IDH1/2 mutant gliomas in TCGA and Rembrandt (Fig. $1 b)$.

Then we tried to explore the prognostic value of FTL in gliomas by using public datasets. 50\% cutoff point was used Kaplan-Meier analysis for FTL expression. Our results showed that glioma patients with higher FTL expression had worse overall survival than those with lower FTL expression in TCGA, CGGA and Rembrandt datasets (Fig. 1g, Figure S1B-C). Then we examined FTL expression on prognosis of HGG and LGG patients. The results showed that patients with high FTL expression had reduced survival time compared to patients with low FTL expression in HGG subgroup (Fig. 1h, Figure S1D-E). Though FTL expression segregated LGG patients into survival groups in CGGA and Rembrandt datasets (Figure S1F-G),FTL expression wasn't significantly associated with survival time in TCGA dataset (Fig. 1i). Moreover, Cox regression analysis revealed that FTL was an independent risk factor for overall survival in patients with glioma $(\mathrm{HR}=1.44,95 \% \mathrm{CI}(1.06-1.95)$, $p=0.02$,Table 1 ).

\section{Hypoxia induced FTL in a HIF-1a dependent manner}

Hypoxia condition is extremely common in glioma tissues and it is a crucial factor that contributes to the aggressive behavior of glioma. In our study, we tried to investigate the relationship between hypoxia and FTL expression in glioma. We used normalized RNAseq in TCGA datasets and found FTL expression significantly correlated multiply hypoxia-related markers, such as HIF2A, VEGFA, CA9 and PGK1(Figure S2A). Then the results of IHC staining showed that FTL was positively correlated with HIF1A in glioma tissues (Fig. 2a-b). Besides, the areas where FTL was highly expressed tended to co-expressed with higher expression of HIF1A (Figure S2B). Hypoxic area in glioma tissues always presented with more necrosis and microvascular proliferation (Mvp). Ivy glioblastoma atlas project (Ivy GAP) is a dataset that contains anatomic and genetic basis of glioblastoma at the cellular and molecular levels. FTL expression was higher in pseudopalisading cells around necrosis (Pan.) and Mvp areas than in other anatomic structures (Fig. 2c). These results indicated that FTL expression significantly associated with hypoxic environment. Then U87 and U251 cells were cultured under a hypoxic growth condition. We found that FTL expression was increased in U87 and U251 cells under hypoxia in a time-dependent manner (Fig. 2d).

To explore the role of hypoxia in regulating FTL expression, we again placed U87 and U251 cells under hypoxia condition. FTL protein level peaked at $24 \mathrm{~h}$ after hypoxia induction both in U87 and U25 cells, which was consistent with the pattern of increased HIF1A protein levels (Fig. 2e). However, HIF-2 $\alpha$ expression was induced 


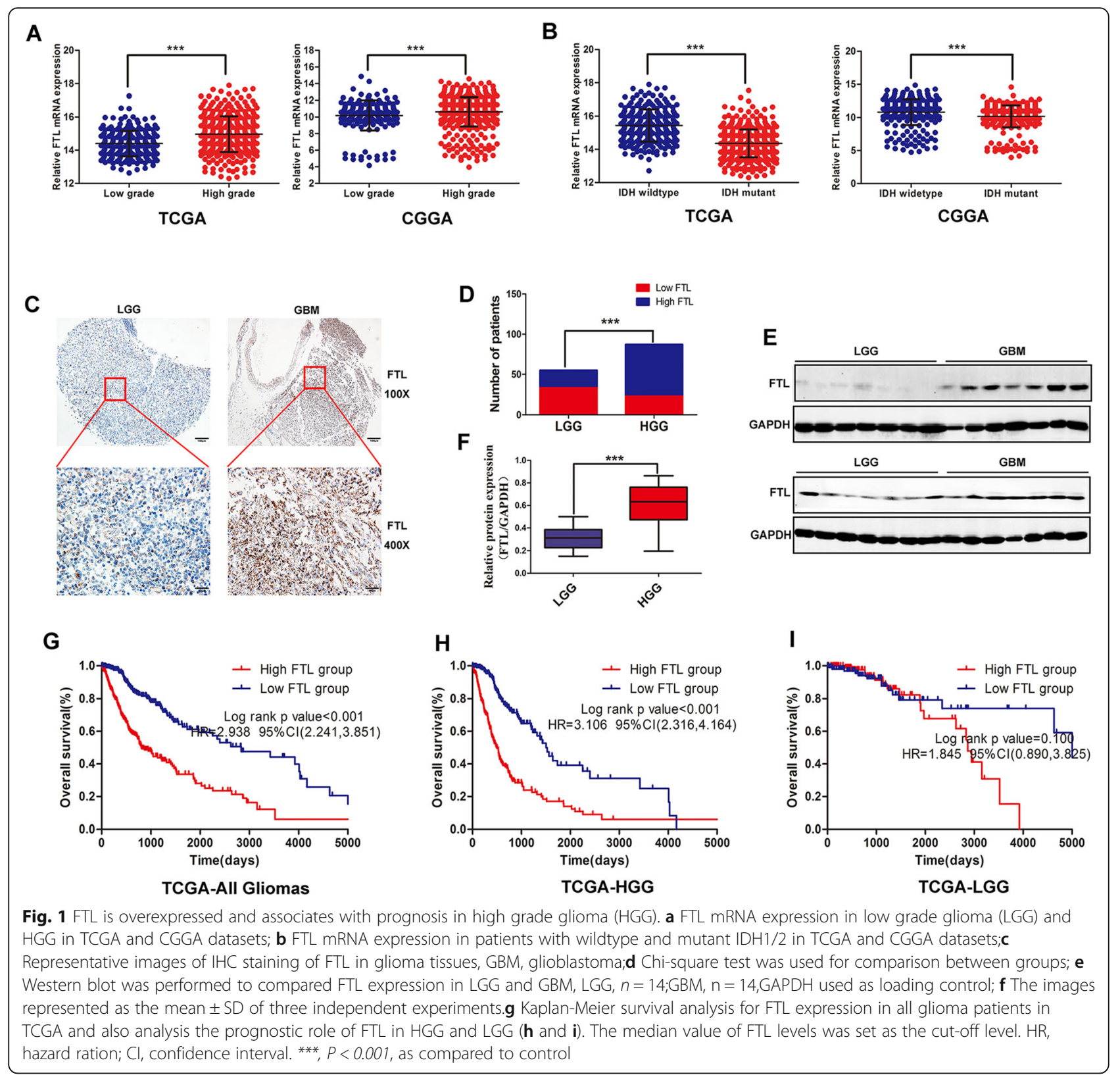

Table 1 Univariate analysis and multivariate COX analysis of clinical prognostic parameters in TCGA dataset

\begin{tabular}{|c|c|c|c|c|}
\hline \multirow[t]{2}{*}{ Variables } & \multicolumn{2}{|c|}{ Univariate Cox regression } & \multicolumn{2}{|c|}{ Multivariate Cox regression } \\
\hline & $\mathrm{HR}(95 \% \mathrm{Cl})$ & $P$ value & $\mathrm{HR}(95 \% \mathrm{Cl})$ & $P$ value \\
\hline $\begin{array}{l}\text { Age } \\
(\geq 55 y \text { vs }<55 y)\end{array}$ & $5.27(3.95-7.03)$ & $<0.001$ & $2.05(1.49-2.84)$ & $<0.001$ \\
\hline $\begin{array}{l}\text { Gender } \\
\text { (Female vs male) }\end{array}$ & $0.85(0.64-1.11)$ & 0.23 & - & - \\
\hline $\begin{array}{l}\text { WHO Grade } \\
\text { (high vs low) }\end{array}$ & $5.43(.68-8.04)$ & $<0.001$ & $2.23(1.45-3.42)$ & $<0.001$ \\
\hline $\begin{array}{l}\text { IDH status } \\
\text { (Wildtype vs mutant) }\end{array}$ & $10.58(7.77-14.41)$ & $<0.001$ & $5.17(3.54-7.55)$ & $<0.001$ \\
\hline $\begin{array}{l}\text { FTL expression } \\
\text { (High vs Low) }\end{array}$ & $2.87(2.15-3.83)$ & $<0.001$ & $1.44(1.06-1.95)$ & 0.02 \\
\hline
\end{tabular}

TCGA, the cancer genome atlas;WHO,World Health Organization;IDH, Isocitrate dehydrogenase;HR,hazard ration 


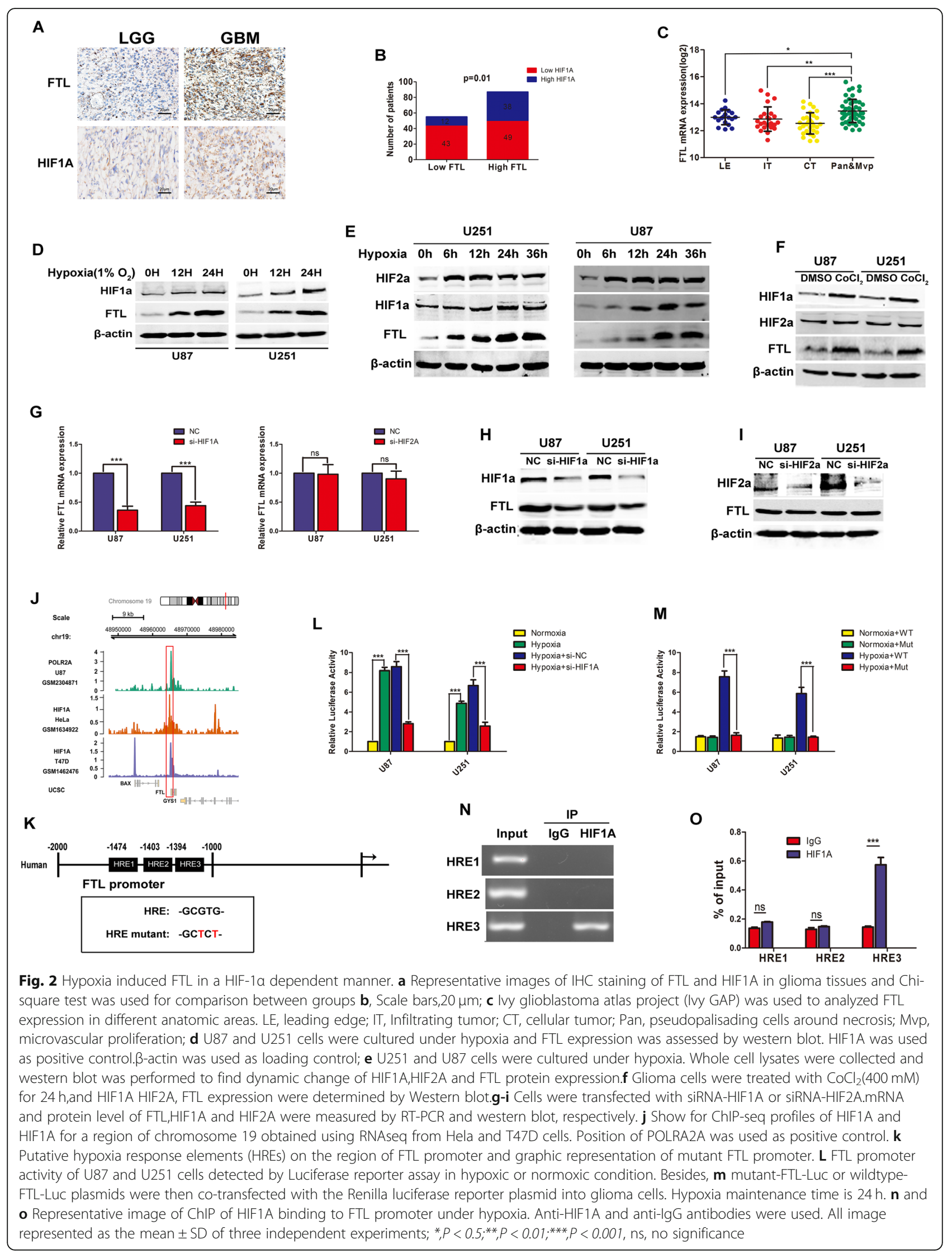


to the peak level at $6 \mathrm{~h}$ after hypoxia induction. To further determine the mechanism by which hypoxia induced FTL expression, we treated U87 and U251 cells with cobalt chloride $\left(\mathrm{CoCl}_{2}, 400 \mathrm{mM}\right)$ for 24 h.The results showed that inhibition HIF1A degradation dramatically promoted the expression of FTL in glioma cells (Fig. 2f). Next, cells were transfected with siRNA-HIF1A or siRNA-HIF2A.The results of RT-PCR and Western blot data showed that knockdown HIF1A could reduce the FTL expression, while inhibiting HIF2A had no effect on FTL expression (Fig. 2g-i).IF staining also confirmed that knockdown HIF1A in glioma cells decreased FTL expression (Figure S2C).

In the promoter region of FTL, we found there were three hypoxia response elements (HREs), and bioinformatics prediction showed that promoter region of FTL may contain HIF1A binding site (Fig. 2j-k). We performed luciferase and ChIP assays to investigate whether HIF1A directly bond to FTL promoter. A full length wildtype FTL luciferase promoter reporter plasmids (FTL-Luc) was constructed. FTL promoter activity was increased in response to hypoxia and knockdown HIF1A in U87 and U251 cells inhibited hypoxia induced FTL promoter activity (Fig. 2l). Then we constructed mutant luciferase plasmid with ablation of HREs on FTL promoter by changing $5^{\prime}$-GCGTG-3' to $5^{\prime}$-GCTCT$3^{\prime}$.Our results showed that transfection of mutant-FTLluc in U87 and U251 cells could dramatically abrogate hypoxia-mediated FTL induction when compared with cells that were transfected with wildtype FTL-Luc (Fig. $2 \mathrm{~m})$.ChIP assay was then performed to further verified direct binding between HIF1A and FTL promoter. In chromatin fractions that were pulled down by antiHIF1A antibody, RT-PCR data showed that only HRE-3 was detected (Figs. 2j, 3n-o).

\section{FTL promoted glioma cells epithelia-mesenchymal transition in vitro and in vivo}

In order to explore the role of FTL in inducing EMT process, we analyzed glioma subtype-specific FTL expression in TCGA and Rembrandt datasets. FTL expression was extremely high in mesenchymal subtype compared with other subtypes (Fig. 3a). Besides, the area Under Curve (AUC) of FTL in predicting mesenchymal subtype of gliomas was 0.908 and 0.845 in TCGA and Rembrandt, respectively (Figure S3A-B). Moreover, we showed that FTL expression positively correlated with multiply EMT-related markers, such as Snail1,Vimentin and Twist1,but negatively correlated with ZEB1(Fig. 3b).

Next, we established stable FTL knockdown glioma cell lines using shRNA. Knockdown FTL obviously altered the morphology of U87 and U251 cells, namely from spindle shape to round shield with less pseudopods (Fig. 3c). Knocking down FTL inhibited the migration and invasion of U87 and U251 cells (Fig. 3d-g). Conversely, overexpression FTL significantly enhanced the migration and invasion of glioma cells (Figure S3C-F). Results of western blot showed that knockdown FTL significantly reduced the expression of vimentin and snaill, but increased E-cadherin expression (Fig. 3g). While overexpression FTL enhanced the expression of EMT markers in U87 and U251 cells (Fig. 3h). Immunofluorescence (IF) staining was then performed to verified our findings and the results showed knockdown FTL obviously altered expression of EMT related proteins of vimentin, snail1 in glioma cells (Figure S3G).

Since hypoxia increased FTL level in glioma, we explored whether FTL mediated hypoxia induced EMT of glioma cells. We used cobalt chloride, a HIF1A inhibitor, to mimic hypoxic conditions. Inhibition of HIF1A obviously enhanced the invasion of glioma cells, while hypoxia-enhanced invasion of glioma cells was blocked by knocking down FTL expression (Fig. 3i-j).What's more, silencing FTL significantly reduced the expression of hypoxia-enhanced EMT markers, such as snial1 and vimentin (Fig. 3k).

Finally, we performed in vivo experiments. Weight of tumors in FTL-shRNA group was dramatically decreased compared with tumors in NC-shRNA group $(0.71 \pm 0.08$ vs $1.17 \pm 0.15, p=0.014$, Fig. 3l-m). Also, the growth of xenografts in FTL-shRNA group were smaller than that of NC-shRNA group (Fig. 3n). Then we used IHC to detect expression of EMT markers and the results showed that inhibition of FTL significantly reduced expression of snail1 and vimentin in vivo (Fig. 3o).

\section{FTL promoted EMT through AKT/GSK3 $\beta / \beta$-catenin signaling}

It is widely recognized that $\beta$-catenin signaling activation induced the expression of multiple EMT markers, such as Snail1,Twist1 and ZEB1 [24]. Our results showed that FTL positively correlated with $\beta$-catenin expression in TCGA (Figure S4A). In our validation cohort, we performed IHC staining and we found patients in high FTL expression group had higher level of $\beta$-catenin when compared with patients in lower FTL expression group (Figure S4B-C).What's more, in glioma tissues with high FTL expression, the accumulation of $\beta$-catenin in the nucleus was more common than in low FTL expression glioma tissues. To further investigate the relationship between FTL and $\beta$-catenin, we performed in vitro experiments. We found that knockdown FTL reduced the expression of $\beta$-catenin, while overexpression FTL increase the expression of $\beta$-catenin (Fig. 4a). In our study, we found the cytoplasm and nucleus fraction of $\beta$ catenin were both decreased after repression of FTL by shRNA in glioma cells (Fig. 4b, e). Since nucleus part of $\beta$-catenin participated in WNT signal transduction, then 


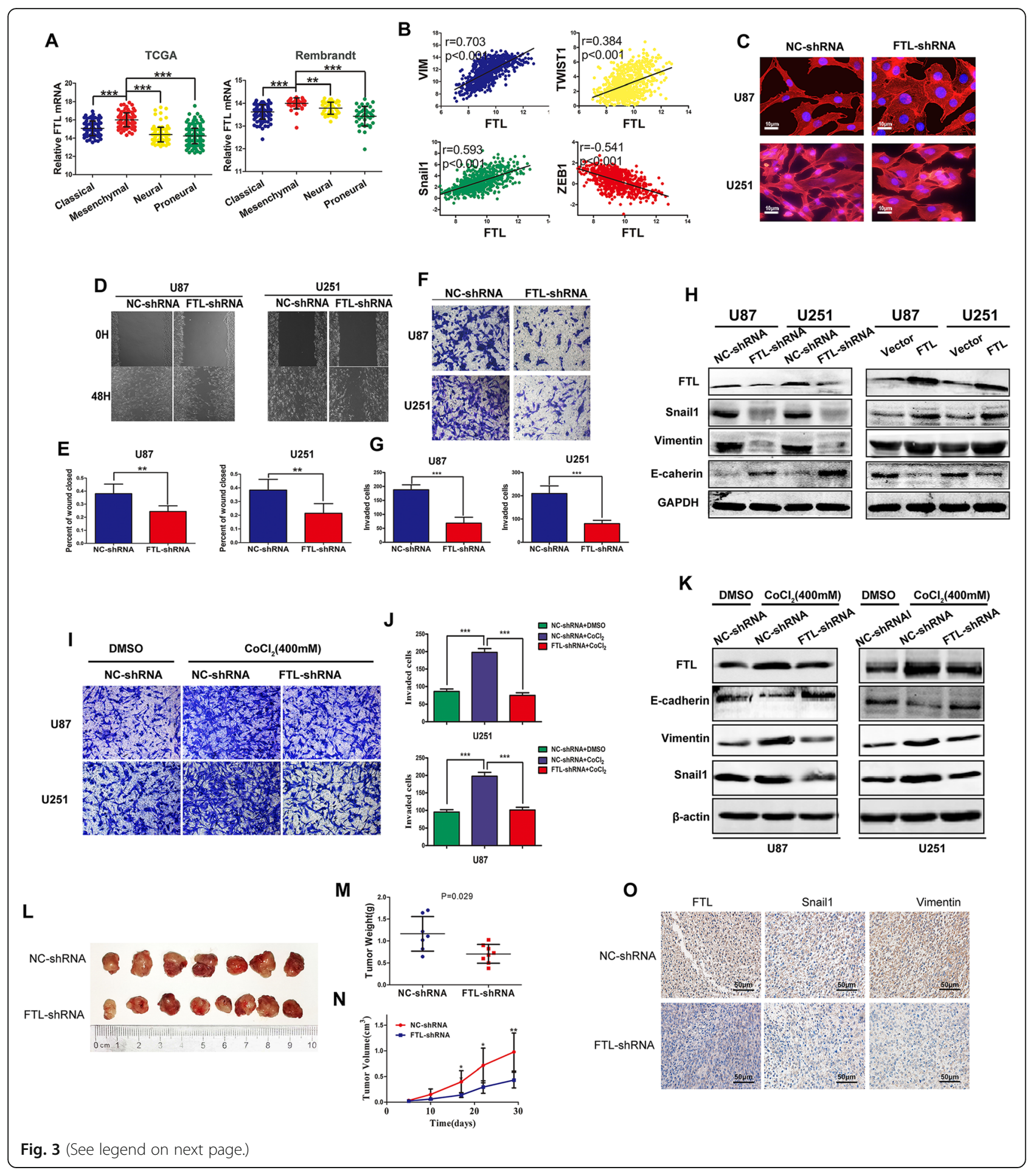


(See figure on previous page.)

Fig. 3 FTL promoted glioma cells epithelia-mesenchymal transition in vitro and in vivo. a Distribution of FTL expression in different glioma molecular subtypes based on RNAseq in TCGA and Rembrandt datasets. b Correlation between FTL with Vimentin, twist1, snail1 and ZEB1 in TCGA. Pearson test was used for correlation analysis. c Representative images of morphology of U87 and U251 cells transfected with NC-shRNA or FTL-shRNA. Cells were staining with phalloidin. Scale bars, $10 \mu \mathrm{m}$. Wound healing (d-e) and transwell assays (f-g) were carried out to detect migration and invasion of glioma cells transfected with NC-shRNA or FTL-shRNA. Wound close percentage was calculated by Image J software (Rawak Software, Inc. Germany).p-values were determined by Student's t-test. $\mathrm{n}=$ three independent experiments. $\mathbf{h}$ Western blot was used to detect expression of snail1,viementin and E-cadherin expression after knocking down or overexpression of FTL in U87 and U251 cells. GAPDH was used as loading control. $\mathbf{i}$-j U87 and U251 cells were treated with DMSO or $\mathrm{CoCl}_{2}(400 \mathrm{mM})$ for $24 \mathrm{~h}$ after transfected with NC-shRNA or FTLshRNA.Transell assay for invasion detection and (k) western blot for E-cadherin,vimentin,snial1 expression measurement. $\beta$-actin was used as loading control. I Fifteen Nude mice were randomly divided into two groups. Images of the xenograft tumors formed in nude mice injected with FTL-shRNA cells and control cells. Tumor volume and tumor weight were calculated $(\mathbf{m}-\mathbf{n})$. o Representative images of IHC staining of FTL,snail1 and vimentin. Scale bars,50 $\mathrm{mm}$. All images represented as the mean \pm SD of three independent experiments; ${ }^{*}, P<0.5, *^{* *}, P<0.01 ;{ }^{* * *}, P<0.001$

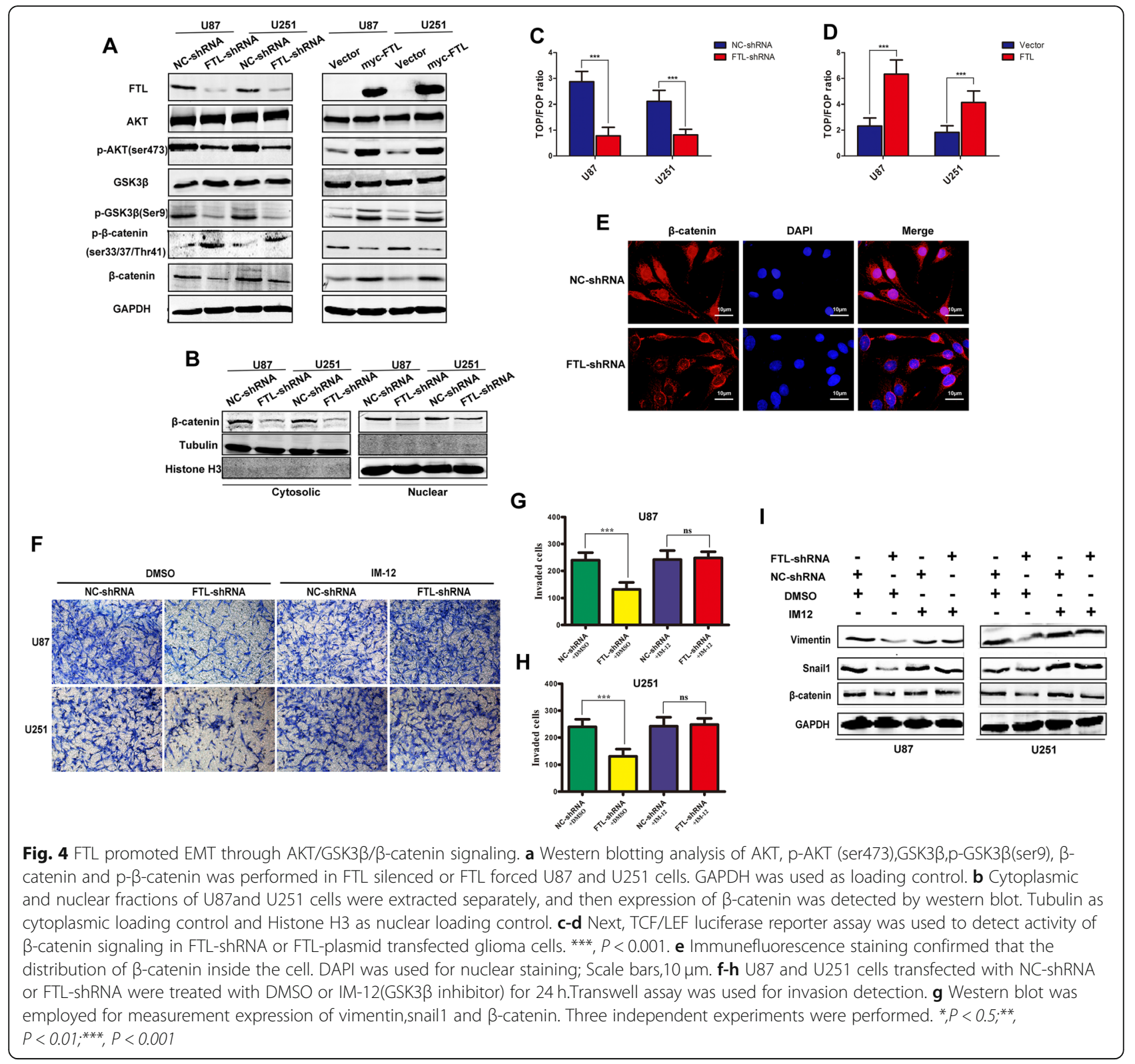


we used luciferase reporter system to detect the activity of $\beta$-catenin signaling. Knockdown FTL reduced the TCF/LEF luciferase activity in U87 and U251 cells (Fig. $4 c-d)$. These results indicated that $\beta$-catenin played crucial role in mediating FTL promoting EMT in glioma cells.

Then we performed WB to detect the expression of AKT,p-AKT,GSK3 $\beta$, p-GSK3 $\beta$ and our results showed that knocking down FTL significantly inhibited the expression of p-AKT (ser473) and p-GSK3 $\beta$ (ser9),but the expression of total AKT and GSK3 $\beta$ had no significant change (Fig. 4a). While overexpression FTL in glioma cells increased p-AKT (ser473) and p-GSK3ß(ser9). We found knockdown FTL in glioma cells increased the phosphorylation of $\beta$-catenin, while forced FTL expression decreased the phosphorylation of $\beta$-catenin (Fig. 4a). Furthermore, we selected a GSK3 $\beta$ inhibitor (IM-12) and $\beta$-catenin overexpression plasmid to complete rescue experiment. IM-12 is a novel GSK3 $\beta$ inhibitor which can increase the activity of $\beta$-catenin. We found that both IM-12 and $\beta$-catenin plasmid could reversed the effects of FTL knockdown on cell invasion in U87 and U251 cells (Fig. 4f-h, Figure S4D-F). Besides, they could both significantly abolished the effect of FTL on the expression of EMT markers, such as vimentin and snail1(Fig. 4i, Figure S4G). Taken together, these results indicated that FTL promoted EMT of glioma cells by regulating $\mathrm{AKT} / \mathrm{GSK} 3 \beta / \beta$-catenin signaling.

\section{FTL enhanced resistance to temozolomide (TMZ) chemotherapy in glioma cells}

Accumulating evidence has shown that EMT phenotype is closely associated with chemotherapy resistance of glioma cells. First, we used the CCK- 8 assay to detect the survival rate of U87 and U251 cells treated with different concentrations of TMZ and the results showed that inhibition of FTL enhanced the efficacy of TMZ and decreased cell survival rate (Fig. 5a). While overexpression of FTL dramatically increased the survival rate of glioma cells treated with different TMZ concentrations (Figure S5A-B). Moreover, inhibition of FTL increased apoptosis of U87 and U251 cells treated with certain TMZ concentrations $(400 \mu \mathrm{M})$ detected by flow cytometry (Fig. $5 b-c$, while overexpression of FTL decreased the apoptosis of glioma cells when treated with TMZ concentrations $(400 \mu \mathrm{M})$ (Figure S5C-E). Given these results, we assumed that FTL could enhanced TMZ resistance and decreased the cytotoxic effect of TMZ therapy on glioma cells. Moreover, we investigated whether hypoxia induced chemo-resistance was mediated by FTL Inhibition of FTL in glioma cells reduced the clone formation number induced by hypoxia (Fig. 5d-e). Besides, Knockdown FTL significantly induced higher apoptosis rate of cells under hypoxic condition compared with control
(Fig. 5f-g). These results indicated that FTL downregulation could diminished, or partially eliminated hypoxia induced chemoresistance.

Next, we performed in vivo experiment. Six nude mice were randomly divided into two groups and received two cycles of intraperitoneal injection of TMZ $(50 \mathrm{mg} /$ $\mathrm{kg} /$ day, 5 day/cycle).FTL stable knockdown U87 cells generated smaller tumors than the cells expressing $\mathrm{NC}$ shRNA under TMZ treatment (Figure S5F). The growth of implanted tumors in mice injected with FTL-shRNA U87 cells were much lower than cells transfected with NC-shRNA (Figure S5G-H). Furthermore, results of IHC staining also revealed that knockdown FTL in U87 cell significantly reduced the MGMT expression and increased cleaved-caspase3 expression (Figure S5I). Our findings were verified by terminal deoxynucleotidyl transferase nick end labeling (TUNEL) and we found rate of TUNEL positive cells in FTL-shRNA groups was much higher than that of NC-shRNA group (Fig. 5h-i). These results strongly indicated that FTL enhanced resistance to TMZ chemotherapy in glioma and might be a promising target. Taken together, we demonstrated that hypoxia induced FTL promoted EMT by regulating AKT/GSK3 $\beta / \beta$-catenin signaling, which subsequently enhanced invasion and chemoresistance of glioma cells (Fig. 5j).

\section{FTL is a novel biomarker of response to TMZ in glioma}

Since FTL mediated TMZ-resistance of glioma cells, we tried to investigate whether FTL could be used as a biomarker of TMZ therapeutic-response. We used TCGA datasets to analyze the effect of FTL expression on survival time of glioma patients with different treatments. We found that GBM patients with low FTL expression survived significantly longer than those with high expression if they were treated with TMZ at any time (Fig. 6a). While in patients who were treated with ionizing radiation (IR) alone, FTL expression did not segregate GBM patients into survival groups (Fig. 6b). Consistent with this findings, in LGG patients who expressed high FTL had shorter survival time than those expressed low FTL if they treated with TMZ at any time, whereas FTL expression wasn't significantly associated with survival time of patients who were treated with IR alone (Fig. 6c-d). Considering the relationship between MGMT promoter methylation status and TMZ resistance, we explored the relationship between FTL and MGMT. First, we found that FTL expression was positively correlated with MGMT expression in glioma tissues in three public datasets (TCGA,CGGA and Rembrandt) (Fig. 6e). In TCGA, patients with unmethylated MGMT promoter had higher level of FTL than those with methylated MGMT promoter (Fig. 6f). These 


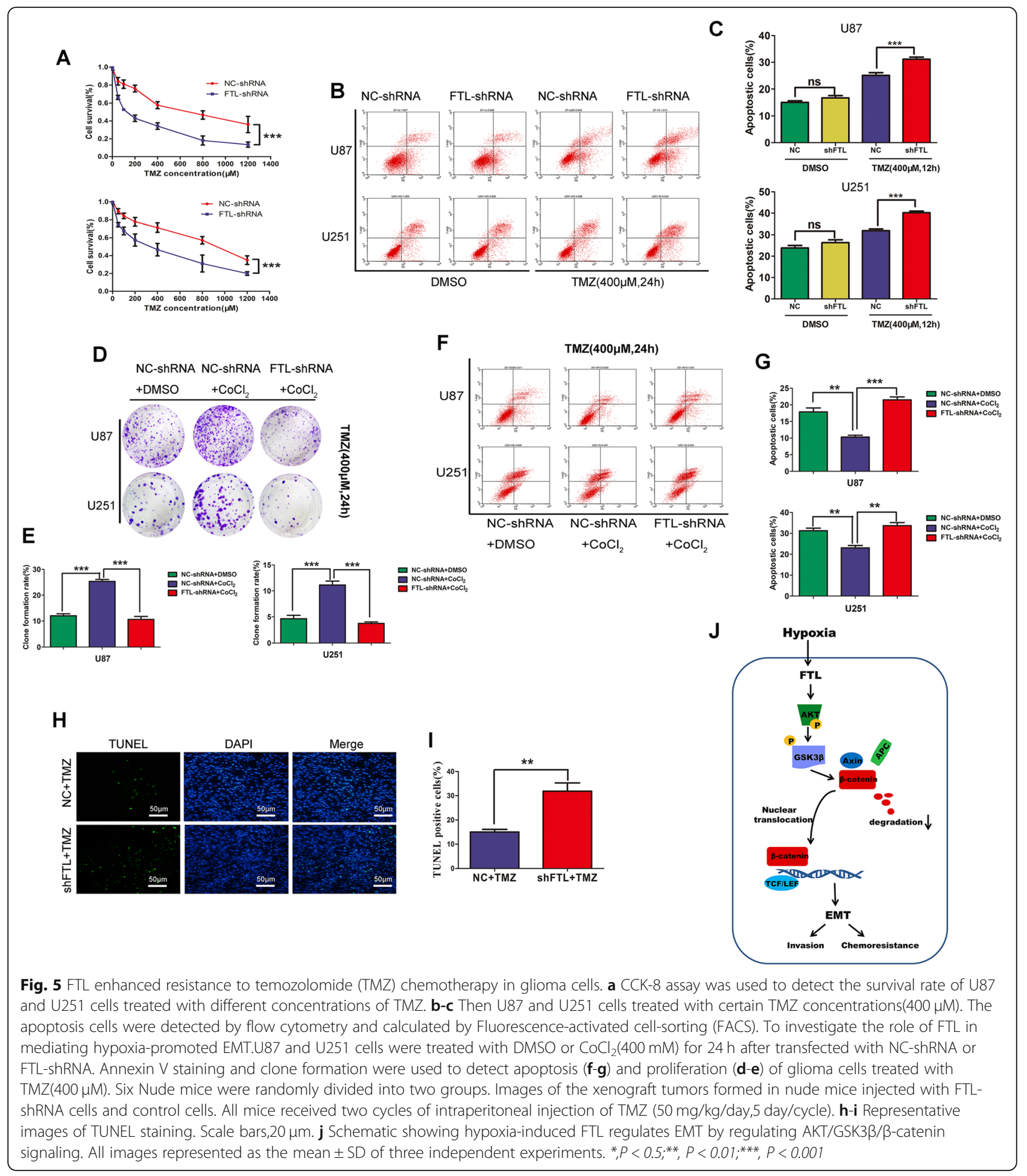

results showed that FTL might be a crucial gene involved in chemoresistance of glioma to TMZ.

Besides, FTL expression only segregate GBM patients into survival groups in tumors with high MGMT promoter methylation, while FTL expression was not informative in tumors with low MGMT promoter methylation (Fig. 6g). Furthermore, in LGG with high MGMT promoter methylation, FTL expression identified distinct survival groups. While FTL expression did not segregate LGG patients with low MGMT promoter methylation into survival groups (Fig. 6h). Our data showed that patients with methylated MGMT who had 


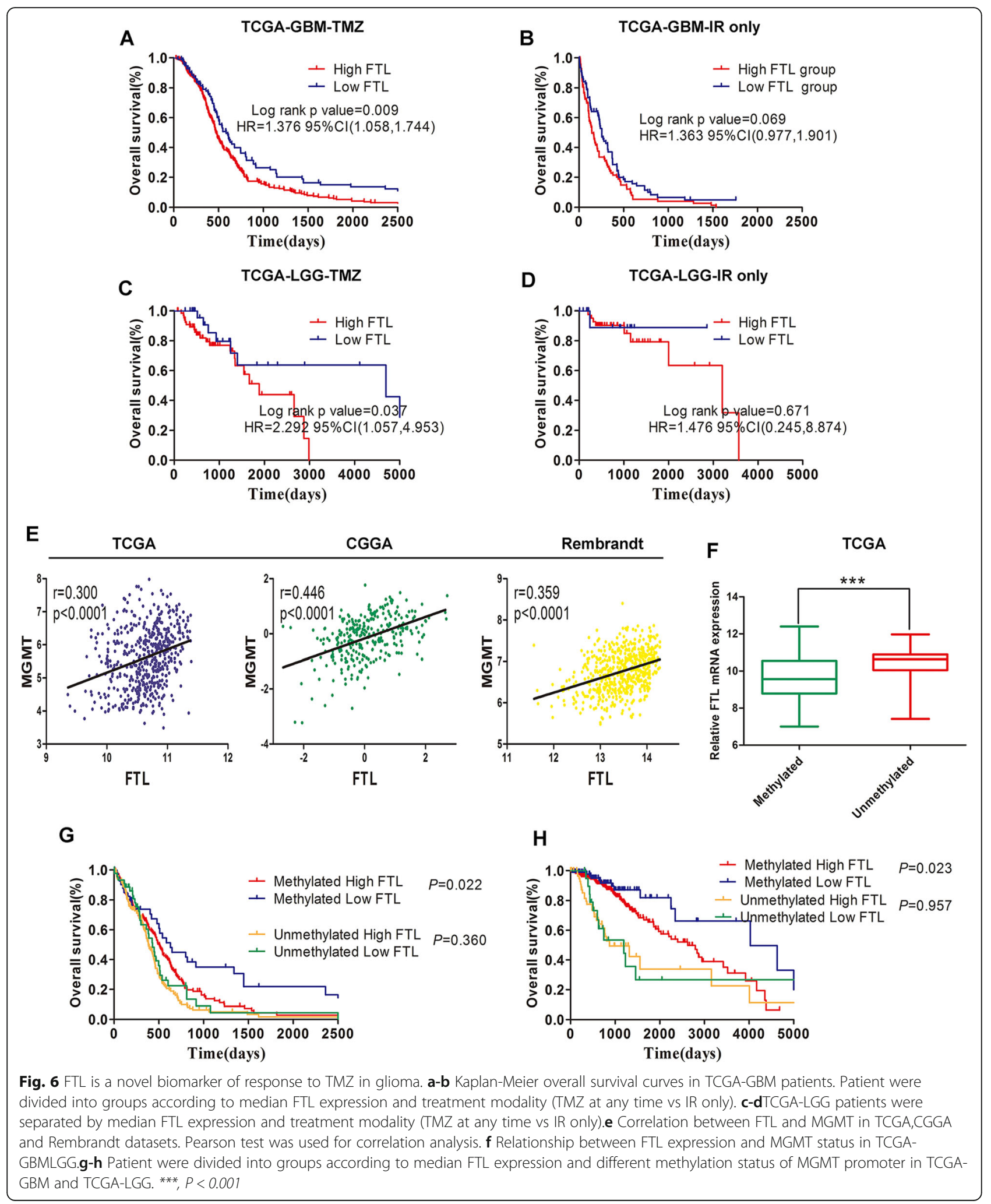


high FTL expression presented similar prognosis with patients with unmethylated MGMT. Taken together, these results suggested that FTL was a novel biomarker in predicting TMZ response in glioma patients.

\section{Discussion}

Hypoxia environment is a fundamental characteristic of malignancy of glioma. The aggressive clinical behavior of glioma are closely related to the tension of oxygen in tumor microenvironment [25]. Hypoxia is considered to be a major driver of malignancy progression and treatment resistance of glioma. Hypoxia inducible factor 1(HIF1) had long been recognized as regulator of the mechanism of hypoxia-promoted progression in glioma [26].. Once cells are under hypoxic condition, HIF-1 $\alpha$ will be gradually accumulated and then translocate to the nucleus. Subsequently, HIF- $1 \alpha$ binds with HIF- $1 \beta$ to form a stable complex which can bind with the hypoxia responsive elements (HREs) in the promoter region of target gene [27]. The promoter of the ferritin gene contains regions of HREs that may interact with HIF$1 \alpha$. Mitochondrial Ferritin (FtMt) is a form of ferritin distribution in mitochondria. FtMt was proved to be an potential target gene of HIF1a,as well as stabilized HIF1a in a hypoxic environment by binding to the HREs that located in the promoter regions of human FTMT gene [20]. In this study, we found a novel hypoxia response gene, FTL, which obviously increased under hypoxia in a time-dependent manner. Besides, there were three HREs in the region of FTL promoter and HIF- $1 \alpha$, not HIF $2 \alpha$ regulates FTL expression by directly binding to HRE-3 in FTL promoter. FTL expression significantly correlated with glioma grade. As it was described before that increasing grade of gliomas correlates with an increase absence of oxygen [4]. So it was reasonable to infer that the high expression of FTL in HGG might be caused by the ubiquitous hypoxic-microenvironment in glioma.

Ferritinwas reported to play crucial role in regulating several solid tumors, including glioma [16, 28, 29]. Glioma cells were considered to undergo EMT during tumorigenesis and progression to higher grade. Recently, several studies uncover closely associated between ferritin and epithelial- mesenchymal transition (EMT). Previous study found that FTL was downregulated in osteosacrcoma (OS) and overexpression FTL in MG-63 cells enhanced the invasion and altered the expression of multiply EMT-related markers [30]. On the contrary, ferritin was evaluated by means of western blot in breast cancer cell lines, and results showed that FTL level was significantly elevated in mesenchymal phenotype cell lines compared with epithelial phenotype [17]. These findings indicate importance of FTL in process of EMT. However, the specific molecular mechanism of how FTL regulates EMT remains unclear. Moreover, authors of another study demonstrated that FTL was a marker of breast tumors with an aggressive phenotype [31]. Consistent with this finding, we found that FTL expression was mainly enriched in mesenchymal subtype and correlated with multiply EMT-related markers in TCGA and CGGA datasets. Also, knocking down FTL dramatically altered glioma cell to blunt morphology and reduced the migration and invasion of glioma cells, as well as alter expression of snail and E-cadherin. The reversal of the EMT process is accompanied by a decrease in the expression of EMT transcription factors and remarkable decline in cell invasiveness [31]. Our study provided solid evidence FTL might be a novel regulator of EMT in glioma. Targeting crucial control mechanisms of EMT could prevent the transformation from epithelial to mesenchymal subtype, which inhibited progression and enhance therapeutic effect of glioma [32].

Accumulating evidence shows that nuclear accumulation of $\beta$-catenin play crucial role in regulating EMT $[33,34]$. Recently, Dong Xiao et al. found that SPHK2, a direct target of miR-708, triggered a cascade of signals leading to the activation of Akt pathway and the phosphorylation of GSK-3 $\beta$ and finally to the nuclear translocation of $\beta$-catenin to regulate EMT in glioma cells [35]. The nuclear accumulation of $\beta$-catenin correlated with WHO grades and cytoplasmic- nuclear $\beta$-catenin was an independent prognostic factors in glioma [36].FTL positively correlated with $\beta$-catenin and the nuclear accumulation of $\beta$-catenin was more common in glioma tissues with high FTL expression. Therefore it was reasonable to infer that FTL may have a certain relationship with $\beta$-catenin. We found that knocking down FTL in glioma cells dramatically reduced nucleus accumulation of $\beta$-catenin and dramatical decrease of activity of $\beta$-catenin signaling detected by Luciferase reporter system. The nuclear translocation of $\beta$-catenin bond with TCF/LEF transcription factors to induce the expression of vimentin and snaill, and subsequently activated the EMT process [37]. In our study, inhibition of FTL inactivated AKT by phosphorylation (ser473) and decrease of the phosphorylation level of GSK3 $\beta$ (ser9). Generally, inactive GSK3 $\beta$, together with Axin, adenomatous polyposis coli (APC), casein kinase 1 (CK1) stabilized $\beta$-catenin which subsequently resulted in nucleus translocation of $\beta$-catenin. Therefore, we inferred that FTL was a regulator of AKT/GSK3 $\beta / \beta$-catenin signaling. Using IM-12 or CTNNB1 plasmid significant reversed the oncogenic function of FTL in mediating EMT in glioma, which strongly indicated that FTL promoted EMT by regulating AKT/GSK3 $\beta / \beta$-catenin signaling.

Interaction between hypoxia and EMT was mediated by multiply genes and pathways. HIF1A might directly induced the expression of TWIST which promoted EMT by interacting with Ring1B and EZH2. However, 
multiply genes might as crucial mediators by which hypoxia induced EMT. Previous study revealed that FAT1 positively correlated with multiply hypoxia related genes and it was a potent regulator of EMT both via or independent of HIFla in glioblastoma [38]. We found that hypoxia enhanced the invasion of glioma cells, while inhibition FTL in glioma cells could mostly eliminate hypoxia-promoted invasion. Consistent with these findings, expressions of EMT-related markers were also obviously altered. Overall, our study revealed that HIF1A directly bond with HRE-3 in the region of FTL promoter to enhance its expression and FTL might act as a crucial gene that regulated EMT process of glioma. Targeting FTL in glioma cells could dramatically inhibit EMT induced by hypoxia, which indicated that FTL could be a potential target for therapy.

As a consequence of EMT process, glioma cells gradually become more invasive, and the adhesion between cells and cells is reduced, which makes it easier to infiltrate the adjacent brain tissues or escape from chemo/ radiotherapy [39, 40]. In addition, EMT alter the stem cell characteristics of tumor cells and express more stem cell markers, which facilitates glioma resistance to chemotherapy and more likely to relapse. Hongbo Guo et al. found that miR-203 expressed low in imatinibresistant GBM cells(U87AR,U251AR), and ectopic expression of miR-203 obviously reversed EMT by directly targeting SNAI2, which sensitized glioma cells to chemotherapy [41]. Also the results of another study revealed that miR-140 that targeted CTSB signaling suppressed the mesenchymal transition of GBM and enhanced TMZ cytotoxicity [42]. Previous studies demonstrated that FTL was participate in chemo-resistance of human breast cancer cells and colorectal cancer and inhibition of FTL induced sensitivity of cells to chemotherapy agents [20]. Consistent with these findings, we found thatknocking down FTL significantly inhibited the proliferation and increased apoptosis of glioma cells treated with $\operatorname{TMZ}(400 \mu \mathrm{M})$. Both in vitro and in vivo showed that cells transfected with sh-FTL were more sensitive to TMZ,which resulted in more apoptotic cells. Together, FTL could enhanced TMZ resistance and decreased the cytotoxic effect of TMZ therapy on glioma cells. The possible mechanistic explanations of FTL-mediated TMZ resistance are that FTL may be an important upstream regulatory protein in the process of MGMT methylation. Besides, HIF1A can activate autophagy. It's possible that hypoxia induced FTL may also affect the autophagic degradation of proteins to affect the resistance of TMZ.

The significance of FTL expression was also demonstrated by its correlation with the clinical prognosis of glioma patients. Upregulation of FTL expression in glioma had been found in several studies, but the relationship of FTL expression and prognosis of glioma has not been well documented. Through bioinformatics analysis and in-house cohort validation, we have identified FTL as a novel biomarker of prognosis, as well as response to TMZ in glioma. Considering the correlation between FTL expression and IDH1/2 or subtypes, use of combination molecular analysis containing FTL might provide a more effective method for predicting prognosis of glioma. Moreover, FTL can be secreted into blood by glioma cells, detecting the level of FTL in plasma may predict the prognosis of glioma. This gives us a hint that FTL may become an important indicator in glioma liquid biopsy. Certainly, more clinical research is needed to clarify these issues regarding biomarker of plasma FTL in glioma.

\section{Conclusion}

The present study showed that HIF1A directly bond with HRE-3 in the region of FTL promoter. FTL promoted EMT of glioma by regulating AKT/GSK3 $3 / \beta-$ catenin signaling, which subsequently enhanced invasion and chemoresistance of glioma cells. Thus, we concluded that hypoxia-inducible FTL was a regulator of EMT and acted not only as a prognostic marker but also a novel biomarker of response to TMZ in glioma.

\section{Supplementary information}

Supplementary information accompanies this paper at https://doi.org/10 1186/s13046-020-01641-8.

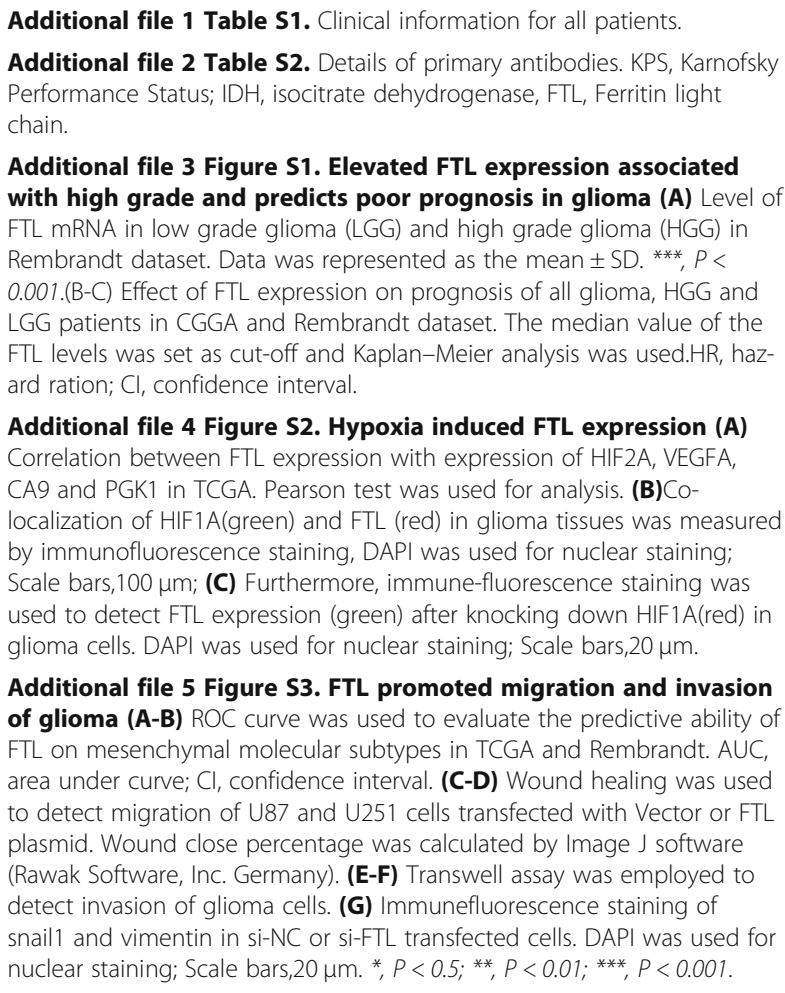

Additional file $\mathbf{1}$ Table S1. Clinical information for all patients.

Additional file $\mathbf{2}$ Table S2. Details of primary antibodies. KPS, Karnofsky Performance Status; IDH, isocitrate dehydrogenase, FTL, Ferritin light chain.

Additional file 3 Figure S1. Elevated FTL expression associated with high grade and predicts poor prognosis in glioma (A) Level of FTL mRNA in low grade glioma (LGG) and high grade glioma (HGG) in Rembrandt dataset. Data was represented as the mean $\pm \mathrm{SD}$. ${ }^{* * *}, P<$ 0.001.(B-C) Effect of FTL expression on prognosis of all glioma, HGG and LGG patients in CGGA and Rembrandt dataset. The median value of the FTL levels was set as cut-off and Kaplan-Meier analysis was used.HR, hazard ration; $\mathrm{Cl}$, confidence interval.

Additional file 4 Figure S2. Hypoxia induced FTL expression (A) Correlation between FTL expression with expression of HIF2A, VEGFA, CA9 and PGK1 in TCGA. Pearson test was used for analysis. (B)Colocalization of HIF1A(green) and FTL (red) in glioma tissues was measured by immunofluorescence staining, DAPI was used for nuclear staining; Scale bars,100 $\mu \mathrm{m}$; (C) Furthermore, immune-fluorescence staining was used to detect FTL expression (green) after knocking down HIF1A(red) in glioma cells. DAPI was used for nuclear staining; Scale bars,20 $\mu \mathrm{m}$.

Additional file 5 Figure S3. FTL promoted migration and invasion of glioma (A-B) ROC curve was used to evaluate the predictive ability of FTL on mesenchymal molecular subtypes in TCGA and Rembrandt. AUC, area under curve; Cl, confidence interval. (C-D) Wound healing was used to detect migration of U87 and U251 cells transfected with Vector or FTL plasmid. Wound close percentage was calculated by Image J software (Rawak Software, Inc. Germany). (E-F) Transwell assay was employed to detect invasion of glioma cells. (G) Immunefluorescence staining of snail 1 and vimentin in si-NC or si-FTL transfected cells. DAPI was used for nuclear staining; Scale bars, $20 \mu \mathrm{m}$. ${ }^{*}, P<0.5$; ${ }^{* *}, P<0.01$; ${ }^{* *}, P<0.001$. 


\section{Additional file 6 Figure S4. Correlation between FTL and $\beta$ -} catenin in glioma tissues. (A) Correlation between FTL and $\beta$-catenin mRNA expression in TCGA. Pearson test was used for correlation analysis. (B-C) Representative images of $\mathrm{HC}$ staining of FTL and $\beta$-catenin in glioma tissues. Scale bars,50 $\mu \mathrm{m}$. Red arrow pointed nuclear accumulation of $\beta$-catenin; Chi-square test was used for comparison between groups; (D-F) U87 and U251 cells transfected with NC-shRNA or FTL-shRNA were then co-transfected with vector or CTNNB1 plasmid. Transwell assay was used for invasion detection. $(G)$. Western blot was employed for detecting expression of Vimentin, Snail1 and $\beta$-catenin. ${ }^{* *}, P<0.01$, ***, $P<0.001$, ns, no significance.

\section{Additional file 7 Figure S5. FTL enhanced TMZ resistance of}

glioma. (A-B) CCK-8 assay was used to detect the survival rate of vector or FTL transfected U87 and U251 cells treated with different concentrations of TMZ.(C-D)U87 and U251 cells treated with certain TMZ concentrations $(400 \mu \mathrm{M})$.(C-E) The apoptosis cells were detected by flow cytometry and calculated by Fluorescence-activated cell-sorting (FACS). Besides, (F) Images of the xenograft tumors formed in nude mice injected with FTL-shRNA cells and control cells. All mice received intraperitoneal injection of TMZ (50 mg/kg/day,5 day/cycle). (G-H) Tumor volume and tumor weight were calculated. (I) Representative images $\mathrm{IHC}$ staining of FTL, MGMT and cleaved-caspased3 in xenograft tumor. Scale bars,50 $\mu \mathrm{m}$. All images represented as the mean \pm SD of three independent experiments. ${ }^{*}, P<0.5 ;{ }^{* *}, P<0.01 ;{ }^{* *}, P<0.001$.

\section{Abbreviations}

ChIP: Chromatin immunoprecipitation; EMT: Epithelia mesenchymal transition; FTL: Ferritin light chain; HGG: High grade glioma; HRE: Hypoxia response elements; HIF: Hypoxia-inducible factor; IHC: Immunochemistry; IDH1/2: Isocitrate dehydrogenase 1/2; LGG: Low grade glioma; TMZ: Temozolomide; WB: Western blot

\section{Acknowledgements}

Not applicable.

\section{Authors' contributions}

Study design and data collection: CQX, LJH, GL, XPF, YJA and CQ; LJH and GL mainly performed the experiments. Data analysis: YFE, XY, GRX and ZN; Manuscript preparation: LJH, GL and CQX. All authors read and approved the final manuscript.

\section{Funding}

This work was supported by the National Natural Science Foundation of China (No. 81572489).

\section{Availability of data and materials}

The data that support the findings of this study are available from the corresponding author upon reasonable request.

\section{Ethics approval and consent to participate}

All patients signed informed consents and this study received the approval of the Ethics Committee of Renmin Hospital of Wuhan University (approved number: 2012LKSZ (010) H). Animal feeding and experimental operations were in line with the guidelines of the Animal Ethics Committee of Wuhan University People's Hospital.

\section{Consent for publication}

Not applicable.

\section{Competing interests}

None.

\section{Author details}

'Department of Neurosurgery, Renmin Hospital of Wuhan University, No.238, jiefang Road, Wuchang District, Wuhan 430060, Hubei Province, China. ${ }^{2}$ Central Laboratory, Renmin Hospital of Wuhan University, Wuhan, China. ${ }^{3}$ Department of Pathology, Renmin Hospital of Wuhan University, Wuhan, China.
Received: 11 March 2020 Accepted: 6 July 2020

Published online: 16 July 2020

\section{References}

1. Ostrom QT, Gittleman H, Truitt G, Boscia A, Kruchko C, Barnholtz-Sloan JS. CBTRUS Statistical Report: Primary Brain and Other Central Nervous System Tumors Diagnosed in the United States in 2011-2015. Neuro Oncol. 2018; 20:iv1-iv86.

2. Ostrom QT, Cote DJ, Ascha M, Kruchko C, Barnholtz-Sloan JS. Adult Glioma incidence and survival by race or ethnicity in the United States from 2000 to 2014. Jama Oncol. 2018:4:1254-62.

3. Verhaak RG, Hoadley KA, Purdom E, Wang V, Qi Y, Wilkerson MD, et al. Integrated genomic analysis identifies clinically relevant subtypes of glioblastoma characterized by abnormalities in PDGFRA, IDH1, EGFR, and NF1. Cancer Cell. 2010;17:98-110.

4. Evans SM, Judy KD, Dunphy I, Jenkins WT, Hwang WT, Nelson PT, et al. Hypoxia is important in the biology and aggression of human glial brain tumors. Clin Cancer Res. 2004;10:8177-84.

5. Miska J, Lee-Chang C, Rashidi A, Muroski ME, Chang AL, Lopez-Rosas A, et al. HIF-1alpha Is a Metabolic Switch between Glycolytic-Driven Migration and Oxidative Phosphorylation-Driven Immunosuppression of Tregs in Glioblastoma. Cell Rep. 2019;27:226-237.e4.

6. Gabriely G, Wheeler MA, Takenaka MC, Quintana FJ. Role of AHR and HIF1alpha in Glioblastoma metabolism. Trends Endocrinol Metab. 2017;28:42836.

7. Luo Z, Bai M, Xiao X, Zhang W, Liu X, Yang X, et al. Silencing of HIF-1alpha enhances the radiation sensitivity of human glioma growth in vitro and in vivo. Neuropharmacology. 2015;89:168-74.

8. Chen W, Cheng X, Wang X, Wang J, Wen X, Xie C, et al. Clinical implications of hypoxia-inducible factor-1alpha and caveolin-1 overexpression in isocitrate dehydrogenase-wild type glioblastoma multiforme. Oncol Lett. 2019;17:2867-73.

9. Liu ZJ, Liu HL, Zhou HC, Wang GC. TIPE2 inhibits hypoxia-induced Wnt/ beta-catenin pathway activation and EMT in Glioma cells. Oncol Res. 2016; 24:255-61.

10. Iwadate Y, Matsutani T, Hirono S, Shinozaki N, Saeki N. Transforming growth factor-beta and stem cell markers are highly expressed around necrotic areas in glioblastoma. J Neuro-Oncol. 2016;129:101-7.

11. Bhuria V, Xing J, Scholta T, Bui KC, Nguyen M, Malek NP, et al. Hypoxia induced sonic hedgehog signaling regulates cancer stemness, epithelial-tomesenchymal transition and invasion in cholangiocarcinoma. Exp Cell Res. 2019;385:111671.

12. Karsy M, Guan J, Jensen R, Huang LE, Colman $H$. The impact of hypoxia and Mesenchymal transition on Glioblastoma pathogenesis and Cancer stem cells regulation. World Neurosurg. 2016;88:222-36.

13. Yu GH, Fu L, Chen J, Wei F, Shi WX. Decreased expression of ferritin light chain in osteosarcoma and its correlation with epithelial-mesenchymal transition. Eur Rev Med Pharmacol Sci. 2018;22:2580-7.

14. Hu Z, Wang L, Han Y, Li F, Zheng A, Xu Y, et al. Ferritin: a potential serum marker for lymph node metastasis in head and neck squamous cell carcinoma. Oncol Lett. 2019;17:314-22.

15. Su Q, Lei T, Zhang M. Association of ferritin with prostate cancer. J Buon. 2017;22:766-70.

16. Rosager AM, Sorensen MD, Dahlrot RH, Hansen S, Schonberg DL, Rich JN, et al. Transferrin receptor-1 and ferritin heavy and light chains in astrocytic brain tumors: expression and prognostic value. PLoS One. 2017;12:e0182954.

17. Jezequel P, Campion L, Spyratos F, Loussouarn D, Campone M, GuerinCharbonnel C, et al. Validation of tumor-associated macrophage ferritin light chain as a prognostic biomarker in node-negative breast cancer tumors: a multicentric 2004 national PHRC study. Int J Cancer. 2012;131:426-37.

18. Kudriavtseva AV, Anedchenko EA, Oparina N, Krasnov GS, Kashkin KN, Dmitriev AA, et al. Expression of FTL and FTH genes encoding ferretin subunits in lung and renal carcinomas. Mol Biol (Mosk). 2009;43:1044-54.

19. Maresca V, Flori E, Cardinali G, Briganti S, Lombardi D, Mileo AM, et al. Ferritin light chain down-modulation generates depigmentation in human metastatic melanoma cells by influencing tyrosinase maturation. J Cell Physiol. 2006;206:843-8.

20. Li Z, Liu J, Chen H, Zhang Y, Shi H, Huang L, et al. Ferritin light chain (FTL) competes with long noncoding RNA Linc00467 for miR-133b binding site to regulate chemoresistance and metastasis of colorectal cancer. Carcinogenesis. 2019;41;467-77. 
21. Smith JJ, O'Brien-Ladner AR, Kaiser CR, Wesselius LJ. Effects of hypoxia and nitric oxide on ferritin content of alveolar cells. J Lab Clin Med. 2003;141: 309-17.

22. Sammarco MC, Ditch $S$, Banerjee A, Grabczyk E. Ferritin $L$ and $H$ subunits are differentially regulated on a post-transcriptional level. J Biol Chem. 2008;283: 4578-87.

23. Louis DN, Perry A, Reifenberger G, von Deimling A, Figarella-Branger D, Cavenee WK, et al. The 2016 World Health Organization classification of tumors of the central nervous system: a summary. Acta Neuropathol. 2016 131:803-20.

24. Evans SM, Judy KD, Dunphy I, Jenkins WT, Nelson PT, Collins R, et al. Comparative measurements of hypoxia in human brain tumors using needle electrodes and EF5 binding. Cancer Res. 2004;64:1886-92.

25. Semenza GL. Targeting HIF-1 for cancer therapy. Nat Rev Cancer. 2003;3: 721-32

26. Balamurugan K. HIF-1 at the crossroads of hypoxia, inflammation, and cancer. Int J Cancer. 2016;138:1058-66.

27. Wu Q, Wu WS, Su L, Zheng X, Wu WY, Santambrogio P, et al. Mitochondrial ferritin is a hypoxia-inducible factor 1alpha-inducible gene that protects from hypoxia-induced cell death in brain. Antioxid Redox Signal. 2019;30: 198-212.

28. Biamonte F, Battaglia AM, Zolea F, Oliveira DM, Aversa I, Santamaria G, et al. Ferritin heavy subunit enhances apoptosis of non-small cell lung cancer cells through modulation of miR-125b/p53 axis. Cell Death Dis. 2018;9:1174.

29. Wu T, Li Y, Liu B, Zhang S, Wu L, Zhu X, et al. Expression of ferritin light chain (FTL) is elevated in Glioblastoma, and FTL silencing inhibits Glioblastoma cell proliferation via the GADD45/JNK pathway. PLoS One. 2016;11:e0149361.

30. Shpyleva SI, Tryndyak VP, Kovalchuk O, Starlard-Davenport A, Chekhun VF, Beland FA, et al. Role of ferritin alterations in human breast cancer cells. Breast Cancer Res Treat. 2011;126:63-71.

31. Lu W, Kang Y. Epithelial-Mesenchymal plasticity in Cancer progression and metastasis. Dev Cell. 2019;49:361-74.

32. Zhang J, Cai H, Sun L, Zhan P, Chen M, Zhang F, et al. LGR5, a novel functional glioma stem cell marker, promotes EMT by activating the Wnt/ beta-catenin pathway and predicts poor survival of glioma patients. J Exp Clin Cancer Res. 2018;37:225.

33. Ge L, Wang H, Xu X, Zhou Z, He J, Peng W, et al. PRMT5 promotes epithelial-mesenchymal transition via EGFR-beta-catenin axis in pancreatic cancer cells. J Cell Mol Med. 2020;24:1969-79.

34. Chen $Y$, Deng $X$, Chen W, Shi P, Lian M, Wang H, et al. Silencing of microRNA-708 promotes cell growth and epithelial-to-mesenchymal transition by activating the SPHK2/AKT/beta-catenin pathway in glioma. Cell Death Dis. 2019;10:448

35. Liu C, Tu Y, Sun X, Jiang J, Jin X, Bo X, et al. Wnt/beta-catenin pathway in human glioma: expression pattern and clinical/prognostic correlations. Clin Exp Med. 2011;11:105-12.

36. He L, Zhou H, Zeng Z, Yao H, Jiang W, Qu H. Wnt/beta-catenin signaling cascade: a promising target for glioma therapy. J Cell Physiol. 2019;234: 2217-28.

37. Srivastava C, Irshad K, Dikshit B, Chattopadhyay P, Sarkar C, Gupta DK, et al. FAT1 modulates EMT and stemness genes expression in hypoxic glioblastoma. Int J Cancer. 2018;142:805-12

38. Aiello NM, Kang Y. Context-dependent EMT programs in cancer metastasis. J Exp Med. 2019;216:1016-26.

39. Liao H, Bai Y, Qiu S, Zheng L, Huang L, Liu T, et al. MiR-203 downregulation is responsible for chemoresistance in human glioblastoma by promoting epithelial-mesenchymal transition via SNAI2. Oncotarget. 2015;6:8914-28.

40. Kahlert UD, Nikkhah G, Maciaczyk J. Epithelial-to-mesenchymal(-like) transition as a relevant molecular event in malignant gliomas. Cancer Lett. 2013:331:131-8

41. Ho KH, Cheng CH, Chou CM, Chen PH, Liu AJ, Lin CW, et al. miR-140 targeting CTSB signaling suppresses the mesenchymal transition and enhances temozolomide cytotoxicity in glioblastoma multiforme. Pharmacol Res. 2019;147:104390

42. Chekhun VF, Lukyanova NY, Burlaka CA, Bezdenezhnykh NA, Shpyleva SI, Tryndyak VP, et al. Iron metabolism disturbances in the MCF-7 human breast cancer cells with acquired resistance to doxorubicin and cisplatin. Int J Oncol. 2013;43:1481-6.

\section{Publisher's Note}

Springer Nature remains neutral with regard to jurisdictional claims in published maps and institutional affiliations.
Ready to submit your research? Choose BMC and benefit from:

- fast, convenient online submission

- thorough peer review by experienced researchers in your field

- rapid publication on acceptance

- support for research data, including large and complex data types

- gold Open Access which fosters wider collaboration and increased citations

- maximum visibility for your research: over $100 \mathrm{M}$ website views per year

At $\mathrm{BMC}$, research is always in progress.

Learn more biomedcentral.com/submissions 